\title{
The Detection of a 3.5-h Period in the Classical Nova Velorum 1999 (V382 Vel) and the Long Term Behavior of the Nova Light Curve
}

\author{
Şölen Balman ${ }^{1,2}$, Alon Retter ${ }^{3}$, Marc Bos ${ }^{4}$
}

\begin{abstract}
We present CCD photometry, light curve and time series analysis of the classical nova V382 Vel (N Vel 1999). The source was observed for 2 nights in 2000, 21 nights in 2001 and 7 nights in 2002 using clear filters. We report the detection of a distinct period in the light curve of the nova $\mathrm{P}=0.146126(18) \mathrm{d}(3.5 \mathrm{~h})$. The period is evident in all data sets, and we interpret it as the binary period of the system. We also measured an increase in the amplitude modulation of the optical light (in magnitude) by more than $55 \%$ from 2000 to 2001 and about $64 \%$ from 2001 to 2002. The pulse profiles in 2001 show deviations from a pure sinusoidal shape which progressively become more sinusoidal by 2002. The main cause of the variations in 2001 and 2002 can be explained with the occultation of the accretion disk by the secondary star. We interpret the observed deviations from a pure sinusoidal shape as additional flux resulting from the aspect variations of the irradiated face of the secondary star.
\end{abstract}

Subject headings: Stars: cataclysmic variables, novae - Individual: V382 Vel binaries: close - accretion, accretion disks - white dwarfs

\section{INTRODUCTION}

A classical nova outburst arises from the explosive ignition of accreted matter (i.e., thermo-nuclear runaway, TNR) in a cataclysmic binary system where a Roche-lobe filling

\footnotetext{
${ }^{1}$ Middle East Technical University, Inönü Bulvarı, Ankara, Turkey, 06531; solen@astroa.physics.metu.edu.tr

${ }^{2}$ Astrophysics Missions Division, Research and Scientific Support Department of ESA, ESTEC, Postbus 299 SCI-SA, Keplerlaan 12201 AZ Noorwijk ZH, The Netherlands

${ }^{3}$ Department of Astronomy and Astrophysics, Pennsylvania State University, 525 Davey Lab., University Park, PA 16802, USA

${ }^{4}$ Mount Molehill Observatory, Auckland, NZ
} 
secondary transfers hydrogen rich material typically via an accretion disk onto the white dwarf (WD) primary. During the outburst, the envelope of the WD expands to $\sim 100$

$\mathrm{R}_{\odot}$ and $10^{-7}$ to $10^{-3} \mathrm{M}_{\odot}$ of material that exceeds the escape velocity is expelled from the system (Shara 1989; Warner 1995; Starrfield 2002). The outburst stage usually lasts from a few months to several years and finally, the system returns to a quiescent state after the outburst.

Nova Vel 1999 (V382 Vel) was discovered on 1999, May 20.6 UT (Lee at al. 1999). It is believed to be an outburst on an ONeMg white dwarf because of the strong Ne line emission detected in the optical wavelengths (Woodward et al. 1999). Spectroscopic observations from 5 to 498 days (after the eruption) indicate that the nova belongs to a broad Fe II spectroscopic class with an absolute magnitude at the optical maximum $\mathrm{M}_{v} \sim-8.9, \mathrm{t}_{2}=6 \mathrm{~d}$ and $t_{3}=10 \mathrm{~d}$ (Della Valle et al. 2002). The early evolution of the nova shows an iron curtain phase and P-Cygni profiles on all the important resonance lines with expansion velocities between 2000-4000 $\mathrm{km} \mathrm{s}^{-1}$ (see Shore et al. 2003 and Della Valle et al. 2002 for the optical and Burwitz et al. 2002 for the X-ray wavelengths). Fragmentation in the ejecta is apparent and the ejected mass is calculated to be $4-5 \times 10^{-4} \mathrm{M}_{\odot}$. The nova is found to be enhanced in $\mathrm{Ne} / \mathrm{Ne}_{\odot}=17( \pm 3), \mathrm{N} / \mathrm{N}_{\odot}=17( \pm 4), \mathrm{C} / \mathrm{C}_{\odot}=0.6( \pm 0.3), \mathrm{Al} / \mathrm{Al}_{\odot}=21( \pm 2)$, and $\mathrm{Mg} / \mathrm{Mg}_{\odot}=2.6( \pm 0.1)$ (Shore et al. 2003). The distance to the nova is estimated as 1.7-2.5 kpc (Della Valle et al. 2002; Shore et al. 2003). In addition, Nova Vel 1999 appeared as a Super Soft X-ray Source (Orio et al. 2002; Ness et al. 2005) and the hard X-ray emission was detected originating from the shock heated material in the shell (Orio et al. 2001; Mukai \& Ishida 2001; Burwitz et al. 2002; Ness et al. 2005)

Bos et al. (2002) discovered a 3.5-h periodicity; 0.14615(1) d together with its second harmonic in the optical light curve of the nova. In this paper, we will elaborate that report, discuss possible physical mechanisms for the variation and try to understand the characteristics of the long term light curve and the detected period.

\section{OBSERVATIONS}

We observed V382 Vel during 2 nights in 2000, 21 nights in 2001 and 7 nights in 2002 through clear filters. Table 1 displays the summary of the observation schedule. The observations in 2000 were obtained with a $0.25 \mathrm{~m}$ (Meade) telescope at the Wharemaru Observatory, New Zealand with a ST6 CCD Camera. The photometry in 2001 and 2002 was carried out using the $0.3 \mathrm{~m}$ telescope at the Molehill Observatory with the ST6b CCD Camera, Auckland, New Zealand. The typical exposure times were $20 \mathrm{~s}$ in 2000, and 45-60 s in 2001 and 2002. Also, three nights in January 2001 were obtained at the $0.75 \mathrm{~m}$ telescope 
of the Sutherland, South African Observatory using a UCT CCD photometer.

Before carrying out the photometric measurements, standard noise reduction was applied to the images and the bias and flat corrections were made. Aperture photometry was performed on the corrected and normalized images. A reference group of three comparison stars, close to the nova, in the same field were used in order to reduce the scintillation effects and derive the relative magnitudes. In Figure 1, we indicate the times of our obseravtions with arrows in a plot of the light curve obtained by the AAVSO covering data from the initial outburst to 2003.

\section{DATA ANALYSIS AND RESULTS}

Using our reduced and calibrated data, we constructed light curves for the given nights in Table 1. A collection of normalized light curves obtained from the longer runs are displayed on Figure 2 for 2000, 2001, and 2002. The observational dates are indicated on each panel of the figure. A modulation of the light curve can be seen in all nights. The typical errors of the data points (in magnitudes) are $0^{m} .03$ for 2000, $0^{m} .014$ for 2001 and $0^{m} .013$ for 2002 as calculated from the standard deviation of the magnitudes of the stars chosen as reference to calculate the differential magnitudes.

We performed Fourier analysis on the time series obtained from the data in order to derive the period of the modulations using the ESO-MIDAS (European Southern Observatory Munich Image Data Analysis System) time series analysis package. Several standard

programs were used like the Scargle Algorithim (Scargle 1982) and Discrete Fourier Analysis using Leahy normalization (Leahy et al. 1983). Figure 3, top panel, shows the power spectrum of the data for the year 2000 where the Scargle algorithm was used for the analysis. The middle and bottom panels in Figure 3 show the power spectrum for the years 2001 and 2002, respectively, derived using similar analysis techniques. The detection limit of a period at the $3 \sigma$ confidence level $(99 \%)$ is a power of 18.2 in the middle panel (2001 data), a power of 13.8 in the top panel (2000 data) and a power of 15.8 in the bottom panel (2002 data) (see also Scargle 1982). Before calculating the power spectra, the individual or consecutive nights were normalized by subtraction of the mean magnitude. In order to correct for the effects of time windows and sampling on power spectra, synthetic constant light curves were created and a few very prominent frequency peaks that appear in these light curves were prewhitened from the data in the analysis. When necessary, the red noise in the lower frequencies was removed by detrending the data using linear or quadratic fits. There was considerable red noise in the power spectra especially for the years 2001 and 2002 . The red noise level at the low frequencies below $10 \mathrm{~d}^{-1}\left(2 \times 10^{-4} \mathrm{~Hz}\right)$ increased by a factor of three from 2000 to 2001 
and 2002 .

We found a prominent period at $\mathrm{P}=0.146126(18) \mathrm{d}$ using the whole data set. The power spectra on Figure 3 show the highest peak at this period and the group of peaks around it are some of the $\pm 1 / 3, \pm 1 / 2, \pm 1$, and \pm 2 day aliases of the detected period. In all the figures except Figure 3, top panel, the second harmonic of $\mathrm{P}$ is present. We did not recover

any other significant and/or persistent period in all the years (2000-2002). The ephemeris for $\mathrm{P}$ determined by fitting a sine curve are :

$\mathrm{T}_{0}=$ HJD $2451966.820( \pm 0.001)+0.146126( \pm 0.000018) \mathrm{E}$

The accumulated error on the period for the entire time span of our optical data is $0.014 \mathrm{~d}$ (about $10 \%$ of one cycle). Figure 4 displays the mean light curves folded on P. The top and bottom panels (among the three panels in the figure) show the folded light curve of the 2001 and 2002 data sets, respectively. The short light curves obtained in 2000 have high statistical errors in comparison with the period modulation amplitude, which gives a large error on the detected period (see Figure 3, top panel). This, in return, yields a high ambiguity in the modulation amplitude and thus, we excluded the mean light curve from Figure 4 . The lines in the middle of the top and bottom panels on Figure 4 show the average variation in the differential magnitude of the three reference stars used in the analysis folded on P (i.e., mean light curve of the reference stars). The number of bins in each panel (on Figure 4) are chosen in accordance with the accumulated error for each data set. The period $\mathrm{P}$ showed an amplitude variation of $<0^{m} .007$ in 2000 . The amplitude of the variations were increased significantly to $0^{m} .014 \pm 0^{m} .003$ in 2001 and to $0^{m} .023 \pm 0^{m} .008$ in 2002 where the shape was almost sinusoidal. The increase in modulation depth was more than $55 \%$ (in magnitude) from 2000 to 2001 and about $64 \%$ from 2001 to 2002 on the average.

\section{DISCUSSION}

We presented 30 nights of data on V382 Vel obtained using clear filters in 2000, 2001 and 2002. We discovered modulations in the light curve of the nova at the period $\mathrm{P}=0.146126$ (18) d with an amplitude of $\Delta m<0.007$ in 2000 and the amplitude increased to $\Delta m=0.014$ in 2001 and to $\Delta m=0.023$ in 2002 . Since the periodicity is persistent and coherent, we propose that this is the binary period of the system.

In general, eclipses or occultation effects are detected from novae in outburst and in quiescence (Leibowitz et al. 1992; Kato et al. 2004; Shafter et al. 1993, Woudt \& Warner 2001, 2002, 2003). For example, V838 Her (N Her 1991) shows variable eclipse depth that lasts from 2 to 3 hours, whose depth varies between 0.1 and 0.4 magnitues in about 4 month 
(Leibowitz et al. 1992). The eclipse depth in V1494 Aql (N Aql No.2) is reported to change by 10 times from 0.05 to 0.5 magnitudes in one year from July 2000 to July 2001 (Bos et al. 2001).

The increase in the modulation amplidutes detected throughout the 2000-2002 light curves indicates an occultation in the binary system that is becoming more apparent with time. This is also accompanied with the decreasing brightness/cooling of the WD by about 2 magnitudes from 2000 to 2001 and by about 1.5 magnitudes after February 2001 until the end of January 2002 (see Figure 1). We interpret the cause of the variations in 2001 and 2002 as the occultation of an accretion disk by the secondary star. Fading of the nova itself, is due to a decline in the optical radiation of an uneclipsed source. Therefore, in time, the relative contribution of the occulted body to the total optical output of the system increases causing an increase in the observed amplitude of the variations. Augusto \& Diaz (2003) found an increase in the blue continuum of the nova spectra by 565 days after the outburst and interpreted it as an indication of the re-establishment of accretion and presence of an accretion disk in the system. This confirms our findings since our observations in 2001 start about 645 days after the outburst. The existence of the accretion disk (i.e., mass transfer via an accretion disk) is also supported by the increasing level of red noise seen in the power spectra in 2001 and 2002 as mentioned in sec.(3) (see also van Der Klis 2000). In addition, the expanding photosphere of the nova is already below the surface of the critical lobe of the WD by 2001 since the X-ray turn-off is between December 1999 and February 2000 (Ness et al. 2005). Moreover, the nova/nova shell is already in the nebular stage by the beginning of our optical observations, thus there can not have been any obscuration originating from the ejected material and/or circumbinary medium.

After a nova explosion, the hot WDs may heat and irradiate their cooler companions once they become Super Soft X-ray Sources (SSS) in the course of their evolution (e.g, V1974 Cyg: DeYoung \& Schmidt 1994; also, GQ Mus: Diaz et al. 1995; V1494 Aql: Hachisu et al. 2004). The orbital period of novae can be detected as a result of the aspect variations of the secondary due to heating from the hot WD (Kovetz, Prialnik, \& Shara 1988) and the asymmetry in the pulse profiles could be produced once the shape of the secondary is of a tear drop model. The irradiation effects in classical novae can also be detected long after the outburst stage (e.g., V1500 Cyg: Sommers \& Naylor 1999; DM Gem: Retter et al. 1999). As mentioned before, V382 Vel was detected as an SSS and found to turn off the H burning between December 1999 and February 2000 (about eight months after the outburst). Our observations start in 2000 May 30 and June 2 where the stellar remnant was still hot and the soft-flux was declining in the X-ray wavelengths (Burwitz et al. 2002; Ness et al. 2005). As a result, we expect to see some irradiation effects in this system. 
The orbital variations in V1974 Cyg were $\sim 0^{m} .1$ in the $I$ band and $<0^{m} .05$ in the $V$ band about the time the H-burning turned-off in 1993 (the WD temperature was about $3-4 \times 10^{5} \mathrm{~K}$; Balman et al. 1998). The modulation depth in the I band decreased to about 0.025 magnitudes in 1994 (one year later, Retter et al. 1997) where the remnant WD was no longer detectable in the X-ray wavelengths. Another classical nova detected as an SSS showing irradiation effects, was GQ Mus (the WD temperature was about $5.1 \times 10^{5} \mathrm{~K}$ in 1992 ; Balman \& Krautter 2001 and references therein). The optical light curve showed modulation at the orbital period with an amplitude of 0.33 magnitudes in 1990 that decreased to 0.05 magnitudes in 1994 while the detected asymmetric profile of 1990 changed to a symmetric flat-topped modulation in 1994 (Diaz \& Steiner 1994; Diaz et al. 1995). The optical pulse profile of GQ Mus in 1990 resembles the profile of V1974 Cyg in 1993 and V2275 Cyg in 2003 (N Cyg 2001 No. 2) where the first two are novae with orbital periods below the period gap and the latter a nova with an orbital period of about 7.6 hours (Balman et al. 2003; Balman et al. 2005). The pulse shapes of the orbital modulation of V2275 Cyg changed significantly from 2002 to 2003 and the modulation amplitude decreased (at the orbital period) from 0.42 magnitudes in 2002 to 0.22 magnitudes in 2003, while the irradiated face of the secondary cooled in time (Balman et al. 2005). This source was not observed in the X-rays, thus an SSS phase is not confirmed.

A close inspection of the 2001 light curve of V382 Vel reveals that the modulation shape shows deviations from a pure sinusoidal. Therefore, we constructed a synthetic sine wave using the orbital frequency and subtracted this from the 2001 light curve which would be equivalent to the removal of the variations as a result of the occultation of the disk. The middle panel of Figure 4 shows the residual emission in the light curve of V382 Vel folded on the orbital period. There is additional flux at phases about $\Phi \simeq 0.10-0.15$ and 0.5-0.6. These phases correspond to the position of the secondary at about $90^{\circ}$ to our line of sight. Thus, these humps can possibly be explained by the emission from the irradiated side of the secondary (eg., at the vicinity of L1, the lagrangian point) falling into our line of sight. The observed excess dip at about the orbital minimum (about $\Phi \simeq 0.8-0.9$ ) is, then could be caused by the self-occultation of the irradiated zone by the secondary star when the star is between the WD and the observer. As time progresses, much less emission from the irradiated secondary intervenes with the modulation depths (due to occultation of the accretion disk) in 2002 resulting in a more sinusoidal pulse shape. The scenario described here requires a relatively high inclination angle for the system.

We did not recover any significant superhump period in the light curve of V382 Vel given the time span of our observations that extends to about 985 days after outburst. Superhumps are QPOs caused by tidal instabilities in accreting binary systems with $\mathrm{M}_{2} / \mathrm{M}_{1}<0.35 \pm 0.02$ (see Patterson et al. 2005 for a general review; Nelemans 2005 for AM CVns). In classical 
novae superhumps can be used as probes to study how accretion is reestablished after the outburst. Several systems show such QPOs as early as two and a half months after the outburst (V4633 Sgr, Lipkin et al. 2001) or even two years after the outburst like V1974 Cyg (Retter et al. 1997).

We checked the possibility of any change in the detected binary period. This could have revealed a system that was recoiling to its original size after the initial eruption with a slight expansion in the binary separation. The entire light curve were separated into six consecutive observations and the period was calculated for each set. The data were consistent with a constant line and no period derivative.

\section{SUMMARY AND CONCLUSIONS}

We have detected a consistent and coherent periodicity $\mathrm{P}=0.146126(18)$ in the optical light curve of the classical nova V382 Vel using the data obtained in the years 2000 (two nights), 2001 (21 nights) and 2002 (seven nights). The observations were conducted at the Wharemaru Observatory (2000; 0.25m telescope) and Molehill Observatory (2000-2001; 0.3m telescope) in New Zealand. We interpret this variation as the binary period of the underlying system. We conclude that the cause of variations is the occultation of an accretion disk by the secondary star. We detect increasing modulation amplitudes as the nova itself cools off in time in the optical wavelengths, and the contribution of light from the occulted disk into the total light curve increases enhancing the amount of variation in the light curve. The increase in the variation amplitude from $2000(\Delta \mathrm{m}<0.007)$ to $2001(\Delta \mathrm{m}=.014 \pm 0.003)$ is larger than $55 \%$ and from 2001 to $2002(\Delta \mathrm{m}=0.023 \pm 0.008)$, it is $64 \%$. We also observe variation of the shape and depth of the mean light curves particularly in 2001. We favor a scenario where variations due to the aspect changes of the irradiated secondary star intervenes with the optical light curve at phases 0.10-0.15, 0.5-0.6 and 0.8-0.9.

The soft X-ray radiation from the WDs in the outburst stage of classical novae could trigger irradiation induced high mass transfer resulting in re-establishment of the accretion disk. Irradiation induced mass transfer cycles occur in compact binaries if the donor star has a shallow convective envelope (fast thermal time scale), or the system has mass transfer driven on a long dynamical timescale, or the photosphere scale height is small (Buning \& Ritter 2004; Ritter, Zhang, \& Kolb 2000). Once the nova is a soft X-ray source it has the potential to irradiate its companion initiating mass transfer (stable/limit cycle) if the basic conditions described above are also met depending on other important parameters like the masses of the two stars, the binary separation, etc. The systems which have orbital periods above $3 \mathrm{hrs}$ tend to form mass transfer limit cycles whereas the systems with periods below 
2.5 hrs show stable mass transfer unless the deriving rate is no larger than a few times the gravitational braking rate (Buning \& Ritter 2004). The H burning phase of classical novae outbursts will be interesting laboratories to study irradiation induced mass transfer phenomenon for CVs. In addition, the hibernation scenario for novae suggests that classical novae remain bright for a few centuries after the eruption because of irradiation induced mass transfer (Shara et al. 1986; Prialnik \& Shara 1986). Nova systems are known to show irradiation effects and/or superhumps in their light curves after the optical decline (see Retter \& Naylor 2000).

The three years of monitoring observations of the classical nova V382 Vel in the optical wavelengths have revealed several important facts on the evolution of classical novae during the outburst stage. It shows that accretion is established and a disk is present early in the evolution (as early as 645 days after the outburst). It also shows evidence that the irradiation of the secondary stars by the hot stellar remnants (WDs) could be a common phenomenon that is apparent in the outburst light curves of the first few years till after the stellar remnants cool off of the X-ray wavelengths. Only few novae have been detected during the SSS phase in the X-rays that lasts a few weeks up to several years after the outburst (eg. less than 9-10 years: Orio 2004) and such observations are difficult to obtain due to the tight schedules of the X-ray satellites. The evolution of the light variations as a result of the aspect changes of the irradiated secondary stars can yield additional indirect proof of the evolution of the hot white dwarfs after the outburst and complement the X-ray observations if systematic long term ground-based observations can be maintained in the optical wavelengths.

The Authors acknowledge the American Association of Variable Stars (AAVSO) for providing the accumulated photometric data of V382 Vel from 1999-2003 (used to construct Figure 1). We thank S. Walker for sending us the 2000 data of V382 Vel. AR was partially supported by a research associate fellowship from Penn State University. Finally, SB would like to acknowledge the support from the Turkish Academy of Sciences with the TUBAGEBIP (distinguished young scientist award) Fellowship.

\section{REFERENCES}

Augusto, A., \& Diaz, M.P. 2003, AJ, 125, 3349

Balman, S., Yilmaz, A., Retter, A., Saygac, T., \& Esenoglu H. 2005, MNRAS, 356, 773

Balman, S., Yilmaz, A., Retter, A., Ak., T., Saygac, T., Esenoglu, H., \& Aslan, Z. 2003, IAU Circ., 8074, 3 
Balman, S., Krautter, J., \& Ögelman, H. 1998, ApJ, 499, 395

Balman, S., \& Krautter, J. 2001, MNRAS, 326, 1441

Burwitz, V. et al. 2002, in Classical Nova Explosions, AIP Conf. Proc., Vol. 637, Margarita Hernanz \& Jordi Jose (eds), p. 377

Buning, A. \& Ritter, H. 2004, A\&A, 423, 281

Bos, M., Retter, A., McCormick, J., \& Velthuis, F. 2001, IAU Circ., 7610, 2

Bos, M., Retter, A., Cook, L., \& Novak, R. 2001, IAU Circ., 7665, 2

Diaz, M. \& Steiner, J.E. 1994, ApJ, 425, 252

Diaz, M.P., Williams, R.E., Phillips, M.M., \& Hamuy, M. 1995, MNRAS, 277, 959

Hachisu, I., Kato, M., Kato, T. 2004, ApJ, 606, L139

Kato, T., Ishiuka R., Uemura M., Starkey D.R., Krajci T. 2004, PASJ, 56, 125

van der Klis, M. 2000, ARA\&A, 38, 717

Kovetz, A., Prialnik D., Shara M.M. 1988, ApJ, 325, 828

Leahy, D.A., Elsner R.F., Weisskopf M.C. 1983, 272, 256

Lee, S., Pearce, A., Gilmore, C., Polland, K.R., Mc Saveney, J.A, Kilmartin, P.M., \& Caldwell, P. 1999, IAU Circ., 7176, 1

Leibowitz, E.M., Mendelson H., Mashal E., Prialnik D., Seitter W.C. 1992, ApJ, 385, L49

Lipkin, Y., Leibowitz, E.M., Retter, A., \& Shemner, O. 2001, MNRAS, 328, 1169

Mukai, K. \& Ishida, M. 2001, ApJ, 551, 1024

Nelemens, G. 2005, in The Astrophysics of Cataclysmic Variables and Related Objects, ASP Conf. Ser. Vol.330, J.M. Hameury and J.P. Lasota (eds), (San Francisco : ASP), p. 27

Ness, J.U., Starrfield, S., Jordan, C. Krautter, J., \& Schmitt, J.H.M.M. 2005, MNRAS, inpress

Orio, M. 2004, in Compact Binaries in the Galaxy and Beyond IAU Colloqium 194, Revisita Mexicana Astronomica y Astrofisica Vol. 20 (series de Conferences), G. Tovmassian \& E. Sion (eds), p. 182

Orio, M. et al. 2001, MNRAS, 326, L13

Orio, M., Parmar, A., Greiner, J., Ogelman, H., Starrfield, S., \& Trussoni, E. 2002, MNRAS, 333, L11

Patterson, J. et al. 2005, PASP, in press (astro-ph/0507371) 
Retter, A., Leibowitz E.M., Ofek E.O. 1997, MNRAS, 286, 745

Retter, A., Leibowitz E.M., \& Naylor T. 1999, MNRAS, 308, 140

Retter, A., \& Naylor T. 2000, MNRAS, 319, 510

Ritter, H., Zhang, Z.Y., \& Kolb, U. 2000, A\&A, 360, 969

Scargle, J.D. 1982, ApJ, 263, 835

Shafter, A.W., Misselt, K.A., \& Veal, J.M. 1993, PASP, 105, 853

Shara, M. 1989, PASP, 101, 5

Shara, M., Livio, M., Moffat, A.F.J., \& Orio, M. 1986, ApJ, 311, 163

Prialnik, D. \& Shara, M. 1986, ApJ, 311, 172

Shore, S. et al. 2003, AJ, 125, 1507

Somers, M.W. \& Naylor T. 1999, A\&A, 352, 563

Starrfield, S. 2002, in Classical Nova Explosions, AIP Conf. Proc., Vol. 637, Margarita Hernanz \& Jordi Jose (eds), p. 89

Warner, B. 1995, Cataclysmic Variable Stars, (Cambridge University Press, Cambridge)

Woodward, C.E. Wooden, D.H., Pina. R.K., \& Fisher R.S. 1999, IAU Circ., 7220, 3

Woudt, P.A. \& Warner, B. 2001, MNRAS, 328, 159

Woudt, P.A. \& Warner, B. 2002, MNRAS, 335, 44

Woudt, P.A. \& Warner, B. 2003, MNRAS, 340, 1011 
Table 1: The Time Table of the Observations (only Clear Filters were used)

\begin{tabular}{cccc}
\hline $\begin{array}{c}\text { UT Date } \\
\text { (ddmmyy })\end{array}$ & $\begin{array}{c}\text { Time of Start } \\
(\text { JD-2451000) }\end{array}$ & $\begin{array}{c}\text { Run } \\
\text { Time }(\mathrm{h})\end{array}$ & $\begin{array}{c}\text { Number of } \\
\text { Frames }\end{array}$ \\
\hline 300500 & 694.86480 & 3.6 & 368 \\
020600 & 697.84761 & 3.4 & 322 \\
070101 & 917.60610 & 0.4 & 117 \\
080101 & 918.60250 & 0.4 & 137 \\
110101 & 921.59740 & 0.7 & 239 \\
260201 & 966.83482 & 3.4 & 409 \\
270201 & 967.86559 & 6.1 & 553 \\
010301 & 969.86190 & 5.5 & 365 \\
030301 & 971.86294 & 8.6 & 405 \\
120301 & 980.89287 & 4.7 & 227 \\
150301 & 983.81037 & 4.8 & 216 \\
200301 & 988.86071 & 5.3 & 255 \\
220301 & 990.90068 & 5.4 & 265 \\
250301 & 993.80471 & 7.1 & 351 \\
080401 & 1007.80075 & 4.6 & 214 \\
140401 & 1013.82624 & 8.0 & 200 \\
150401 & 1014.00021 & 3.3 & 339 \\
210401 & 1020.86706 & 6.0 & 266 \\
260401 & 1025.84035 & 6.2 & 313 \\
160501 & 1045.78056 & 6.7 & 338 \\
310501 & 1060.77713 & 6.3 & 304 \\
030601 & 1063.76066 & 6.2 & 309 \\
010102 & 1275.94739 & 5.3 & 246 \\
020102 & 1276.89827 & 6.5 & 315 \\
060102 & 1280.96234 & 2.5 & 157 \\
070102 & 1281.86860 & 7.4 & 444 \\
200102 & 1294.87748 & 5.1 & 307 \\
210102 & 1295.86859 & 4.1 & 248 \\
220102 & 1296.86293 & 5.3 & 318 \\
& & &
\end{tabular}


Fig. 1. - The AAVSO Lightcurve of V382 Vel obtained from the initial outburst in May 1999 until 2003. The arrows indicate the observation times on Table 1.

Fig. 2. - The figure presents the normalized differential light curve of V382 Vel observed in 2000-2002. The observational dates are noted on each panel. The data obtained in 2000 are taken with the $0.25 \mathrm{~m}$ telescope at the Wharemaru Observatory, New Zealand. The rest of the data are taken with the $0.3 \mathrm{~m}$ Telescope, Auckland, New Zealand. Only clear filters are used. The epoch of the observations are noted on the $\mathrm{x}$ axis. The error bars of the data points are about $0^{m} .03,0^{m} .014$, and $0^{m} .013$ for the years 2000, 2001, and 2002, respectively. The scale of the $\mathrm{x}$ and $\mathrm{y}$ axes are fixed throughout the figure for better comparison.

Fig. 3. - The power spectrum of V382 Vel, derived from the 2000 data set (top panel), the 2001 data set (middle panel), and the 2002 data set (bottom panel). The Scargle algorithm is used for the analyses. The new period is indicated with $\mathrm{P}$ and its significant second harmonic is also noted.

Fig. 4. - The mean light curves of the 2001 and 2002 data sets, folded on the period $(\mathrm{P}=0.146126(18) \mathrm{d})$ are presented at the top, and bottom panels, respectively. The middle panel shows the excess emission in phase once the binary period is prewhitened from the light curve of 2001. The lines in the middle of the three panels show the average variation in the differential magnitude of the three reference stars (used in the analysis) folded on the binary period $(\mathrm{P}=0.146126(18) \mathrm{d}$ ). The first data point in time (start mid-HJD in 2000) is taken as the reference for the three mean light curves and a grouping (averaged over) of 25 phase bins (top), 20 phase bins (middle) and 20 phase bins (bottom) are used for the folding process. 


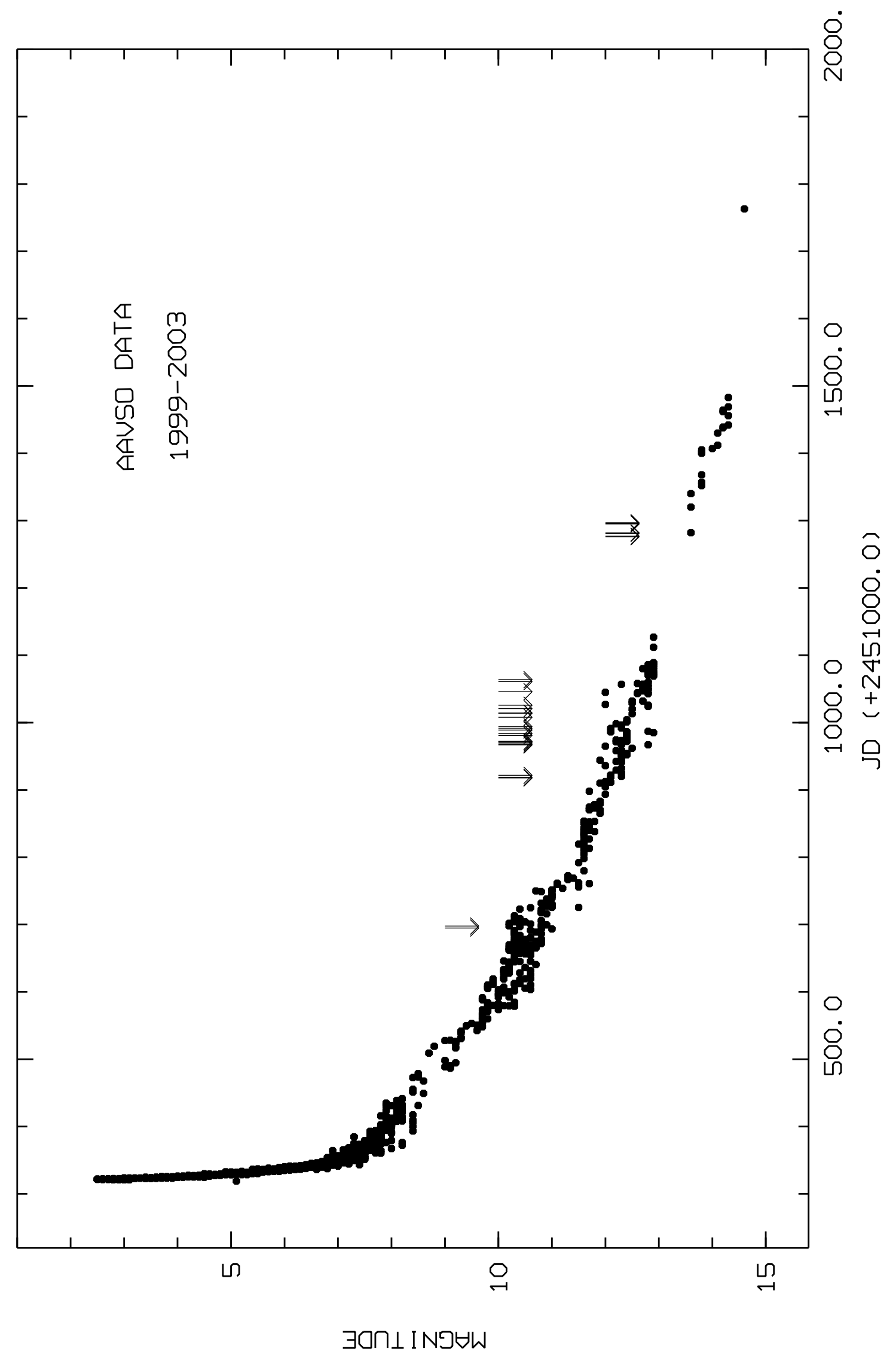



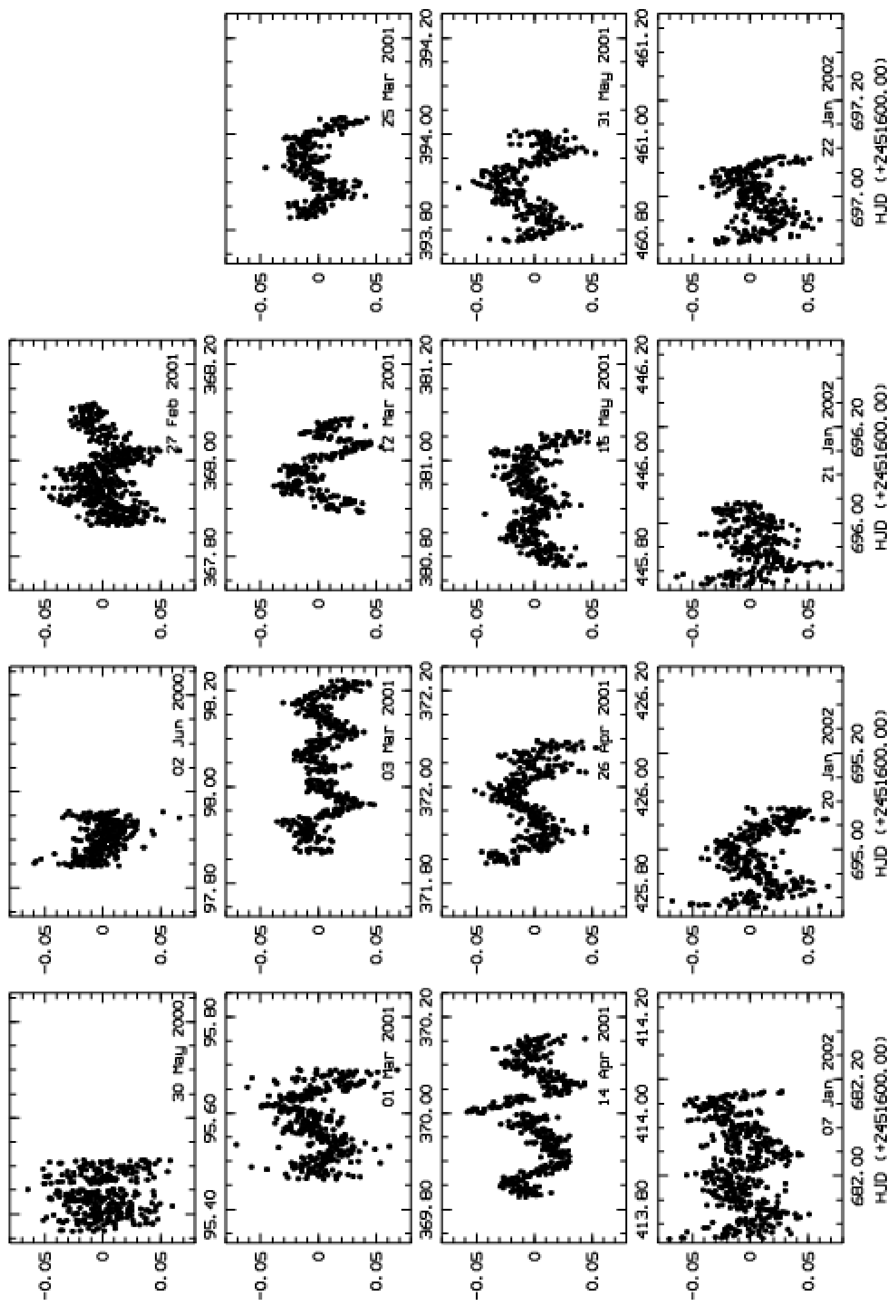

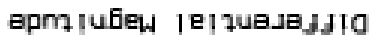

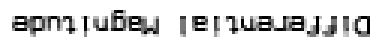



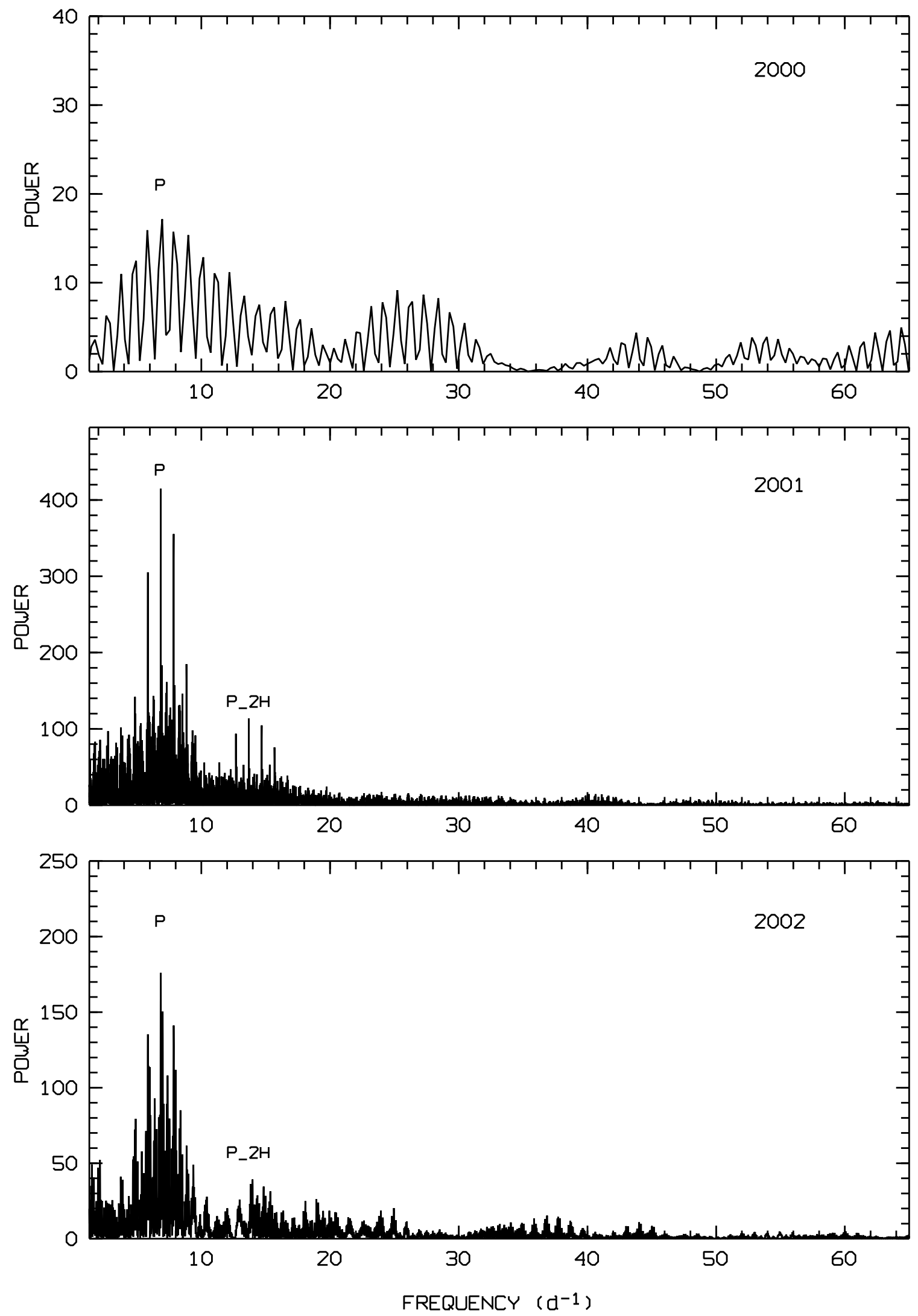

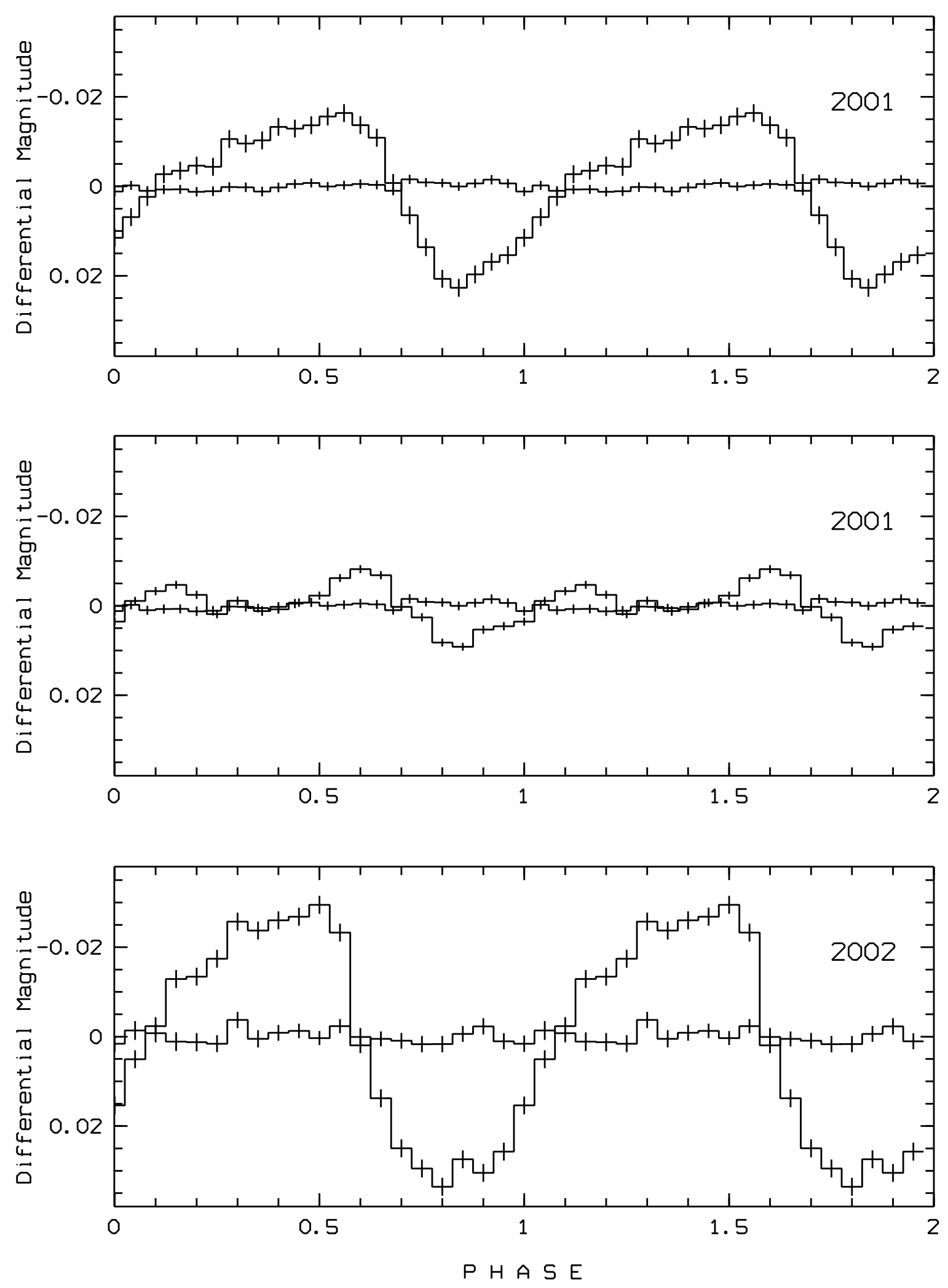